\title{
Identification of bone and liver metastases from breast cancer by measurement of plasma alkaline phosphatase isoenzyme activity
}

\author{
P D MAYNE, ${ }^{*}$ S THAKRAR, * S B ROSALKI, ${ }^{*}$ A Y FOO, * S PARBHOO \\ From the Department of *Chemical Pathology and Human Metabolism, and the $†$ Academic Department of \\ Surgery, Royal Free Hospital and School of Medicine, London
}

SUMMARY Plasma alkaline phosphatase isoenzyme activities were determined in patients with breast cancer to diagnose and monitor bone and liver metastases. Bone alkaline phosphatase activity was increased in 21 of 50 patients $(42 \%)$ with radiologically confirmed bone metastases, while total alkaline phosphatase activity was increased in only 10 of $50(20 \%)$; liver alkaline phosphatase activity was raised in 12 of 25 patients $(48 \%)$ with liver metastases. All patients with liver metastases had bone metastases. Bone alkaline phosphatase activity was significantly higher in patients with symptomatic bone disease. Isoenzyme determination provided additional information that would have changed patient management in five of 20 patients who were monitored serially. Measurement of alkaline phosphatase isoenzyme activity, though less sensitive than imaging procedures, can assist in screening for, and in early detection of, a high proportion of bone and liver metastases, and can provide useful objective evidence of their response to treatment.

More than $50 \%$ of patients with breast cancer develop overt bone metastases and many of these subsequently develop liver metastases. ${ }^{1}$ Survival from breast cancer may be improved by early detection of metastases, as their treatment is usually reserved for symptomatic or progressive disease. The detection of micro or occult metastases is less important as, at present, this finding rarely changes treatment. Bone scintigraphy is a sensitive diagnostic procedure for the detection of overt bone disorders but it is not specific for metastases, and a positive scintigram may require confirmation by radiographical skeletal survey. $^{2}$ These investigations, when performed during follow up to monitor disease, are costly, time consuming, and entail a radiation hazard. Perez et $a l^{3}$ claimed that it is possible to identify $95 \%$ of patients who develop bone metastases by clinical examination, chest $x$-ray, and measurement of plasma total alkaline phosphatase (ALP, EC 3.1.3.1) and $\gamma$-glutamyltransferase (GGT, EC 2.3.2.2) activities. Although easy to perform and inexpensive, neither of the biochemical investigations unequivocally identifies the site of metastases deposits. Increased plasma total alkaline phosphatase activity can result

Accepted for publication 27 October 1986 from either bone or liver secondaries, and though an increase in $\gamma$-glutamyltransferase activity generally indicates liver pathology, extrahepatic malignant disease may give rise to this abnormality. ${ }^{4}$

Recent improvement in plasma alkaline phosphatase isoenzyme separation by affinity electrophoresis with wheat germ lectin permits clear separation of bone and liver isoenzymes and their quantification ${ }^{5}$ and permits their determination to be carried out readily in the diagnostic laboratory. We therefore assessed the value of measuring plasma alkaline phosphatase isoenzyme activity in the diagnosis and monitoring of patients with breast cancer.

\section{Material and methods}

DETERMINATION OF TOTAL ALKALINE

PHOSPHATASE ACTIVITY

Total alkaline phosphatase activity was determined using 4-nitrophenylphosphate as substrate and diethanolamine as buffer. Gamma-glutamyltransferase activity was determined using $\gamma$-glutamyl-4-nitroanilide as substrate and Tris (hydroxymethylaminomethane-glycylglycine buffer. Both assays were carried out at $30^{\circ} \mathrm{C}$ by the proposed methods of the Association of Clinical Biochemists, ${ }^{6}$ adapted for the 
Abbott ABA 100 Bichromatic Analyser (Abbott Laboratories, Dallas, Texas, USA).

\section{DETERMINATION OF ALKALINE PHOSPHATASE} ISOENZYME ACTIVITY

Alkaline phosphatase isoenzyme fractions were separated by electrophoresis and quantified by densitometry. For the demonstration of biliary alkaline phosphatase, samples $(1 \mu \mathrm{l})$ were electrophoresed on cellulose acetate membrane using Tris-barbitone buffer. Alkaline phosphatase activity was identified by incubating the membrane with a chromogenic enzyme substrate, p-toluidinium 5-bromo-4-chloro-3 indoxyl phosphate (Sigma Chemical Co, Poole, UK). Biliary alkaline phosphatase is absent in health, when present in disease it is well separated from the other isoenzymes and is of $\alpha-1$ globulin mobility.

Bone and liver alkaline phosphatase isoenzymes were separated by affinity electrophoresis, as described by Rosalki and Foo. ${ }^{5}$ The electrophoretic procedure is identical with the above, but the cellulose acetate membrane is presoaked for 20 minutes in Trisbarbitone buffer containing wheat germ lectin (Sigma Chemical Co, Poole, UK) $50 \mathrm{mg} / \mathrm{l}$. Bone and liver fractions were quantified by reflectance densitometric scanning at $600 \mathrm{~nm}$ using a Corning 720 Densitometer (Corning Medical, Medifield, Massachusetts, USA), and their activities were calculated by multiplying the percentage of each fraction by sample total alkaline phosphatase activity.

Reference limits in our laboratory in healthy adult women and in those attending a breast clinic with benign breast disease are as follows: total alkaline phosphatase $(205 \mathrm{U} / \mathrm{l})$; bone alkaline phosphatase (90 U/l); liver alkaline phosphatase (110 U/l); biliary alkaline phosphatase (undetectable); $\gamma$-glutamyltransferase $(25 \mathrm{U} / \mathrm{l})$. The electrophoretic detection limit for alkaline phosphatase isoenzyme activity is between 5 and $10 \mathrm{U} / 1$.

\section{PATIENT SAMPLES}

All patients were attending a single breast clinic for follow up following previous removal of histologically confirmed breast cancer. Bone or liver metastases suspected at clinical examination were confirmed by radiological and scintigraphic examination (for bone) and by ultrasonic or computer tomographic examination (for liver). Patients were classified as asymptomatic or symptomatic with respect to bone metastases. Blood was taken for enzyme and isoenzyme determinations at each follow up clinic visit. The clinical data were analysed independently from the enzyme measurements by one of us (SP).

\section{Initial study}

Three groups of patients were studied: group 1, 65 patients with no clinical or radiological evidence of bone or liver metastases at the time of entry into the study (median age at time of examination 59 years, range 34 to 86 years); group 2, 50 patients with bone metastases (median age 59 years, range 30 to 83 years); and group 3, 25 patients with both bone and liver metastases (median age 55 years, range 32 to 81 years).

\section{Serial study}

Twenty patients were examined at intervals of about four to six weeks over a median time period of 17 months (range seven to 27 months). The median age at which breast cancer was diagnosed was 51 years (range 26 to 76 years) and entry into the study occurred about four years later (median age at entry 56 years, range 31 to 77 years).

\section{Results}

Table 1 shows the test sensitivity, test specificity, predictive value of a positive result, predictive value of negative result and test efficiency ${ }^{7}$ of plasma total and bone alkaline phosphatase activity determination for the detection of bone metastases in patients with breast cancer with bone metastases only (group 2). Table 2 shows the diagnostic performance of plasma total, liver, and biliary alkaline phosphatase and $\gamma$-glutamyltransferase activity for the detection of liver metastases (group 3).

The bone alkaline phosphatase activity was increased in $42 \%$ of patients with breast cancer with only bone metastases, this incidence being more than twice that of total alkaline phosphatase activity $(20 \%)$. There was no significant difference in bone alkaline phosphatase activity between those patients who had either localised or extensive bone disease. Patients with symptoms of bone pain or tenderness $(n=24)$ had a higher plasma bone alkaline phosphatase activity (median $100 \mathrm{U} / \mathrm{l}$, range 13 to 1420

Table 1 Diagnostic value of total and bone ALP for detection of bone metastases from breast cancer

\begin{tabular}{lll}
\hline & $\begin{array}{l}\text { Total ALP } \\
(\%)\end{array}$ & $\begin{array}{l}\text { Bone ALP } \\
(\%)\end{array}$ \\
\hline $\begin{array}{l}\text { Test sensitivity } \\
\begin{array}{l}\text { Test specificity } \\
\text { Predictive value of positive } \\
\text { test (PV +) }\end{array}\end{array}$ & 20 & 42 \\
$\begin{array}{l}\text { Predictive value of negative } \\
\text { test (PV -) }\end{array}$ & 98 & 98 \\
$\begin{array}{l}\text { Efficiency } \\
\text { Patients with bone metastases only (group 2) }\end{array}$ & 95 \\
Patients with no bone metastases (group 1) & $\mathrm{n}$ & $\mathrm{n}=50$ \\
\hline
\end{tabular}

Sensitivity, $\%$ positivity in disease; specificity, $\%$ negativity in non-disease; $\mathbf{P V}+, \%$ of positive test results due to disease; $\mathbf{P V}$-, $\%$ of negative test results due to non-disease; efficiency, $\%$ of correct test results. 
Table 2 Diagnostic value of total, liver, and biliary ALP and GGT for detection of bone metastases from breast cancer

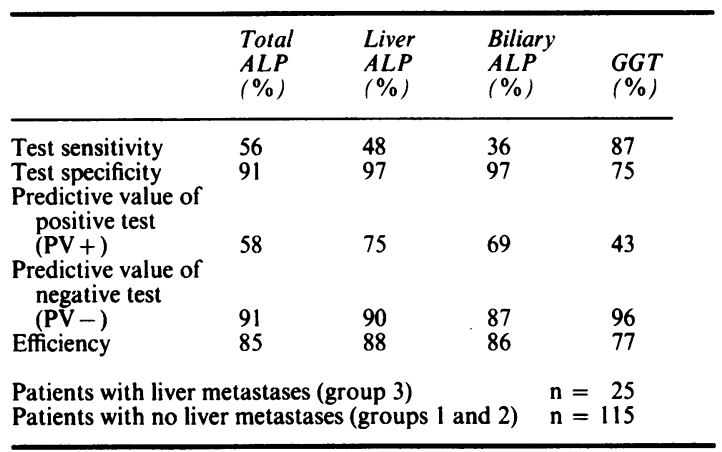

$\mathrm{U} / \mathrm{l})$ compared with that of asymptomatic patients ( $\mathrm{n}=26$, median $38 \mathrm{U} / \mathrm{l}$, range 11 to $161 \mathrm{U} / \mathrm{l}, \mathrm{p}<0.05$ by Mann-Whitney U test) (fig 1 ).

The plasma liver alkaline phosphatase activity was raised in $48 \%$ of the patients in group 3 (bone

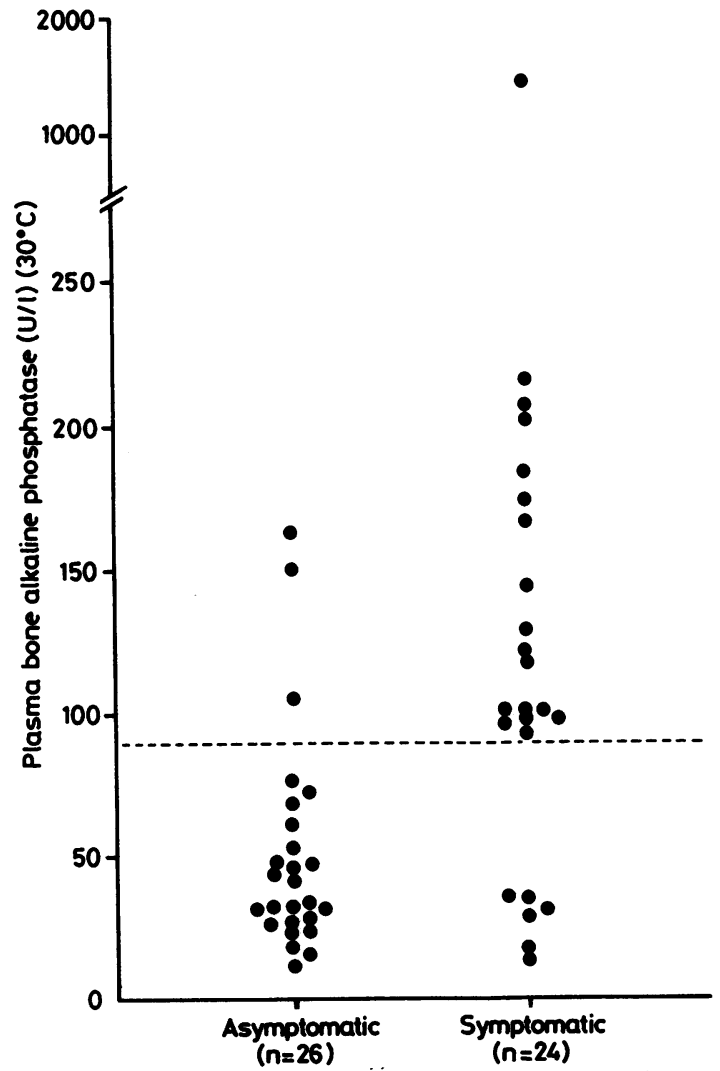

Fig 1 Plasma bone alkaline phosphatase activity in relation to symptoms of bone pain or tenderness in patients with only bone metastases. Plasma bone alkaline phosphatase is significantly higher in symptomatic compared with that in asymptomatic patients ( $p<0.05$ by Mann-Whitney $U$ test).

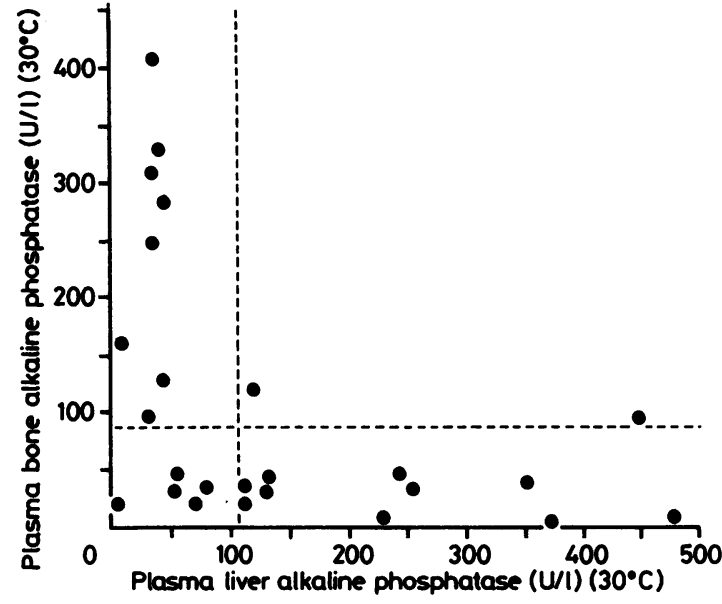

Fig 2 Relation between plasma bone and liver alkaline phosphatase activity in 25 patients with both bone and liver metastases. Note lack of simultaneous increase in liver and bone isoenzymes in all but two patients. Dotted lines indicate upper reference limits.

and liver metastases). Biliary alkaline phosphatase activity was increased in $36 \%$, and total alkaline phosphatase activity in $56 \%$. Plasma $\gamma$-glutamyltransferase activity was increased in $87 \%$ of these patients but was relatively non-specific, being raised in $25 \%$ of patients with breast cancer with no evidence of liver metastases (groups 1 and 2). Only two of the 25 patients in group 3 had both increased plasma bone and liver alkaline phosphatase activity (fig 2). Of the nine patients in whom plasma biliary alkaline phosphatase was detected, seven had an increased liver isoenzyme only, one had a raised bone isoenzyme, and in the remaining patient neither isoenzyme activity was raised.

Seventeen of the 20 patients, who were monitored serially, had bone metastases, and five had liver metastases at the time of entry into the study. One developed bone metastases and four developed liver metastases during the study period. An increase in liver alkaline phosphatase activity gave a lead-in time of between two and nine months in three of these latter four patients. A significant change in enzyme activity was defined as a minimum of two consecutively increasing or decreasing values, each value changing by a minimum of $20 \%$ compared with its predecessor.

Three patients developed "bone flares", shown by an initial increase in uptake on bone scan, at six weeks after the introduction of Tamoxifen treatment. These bone flares were preceded by an earlier acute rise in plasma bone alkaline phosphatase activity. No change in plasma alkaline phosphatase isoenzyme activity accompanied the introduction of any cytotoxic chemotherapy. 


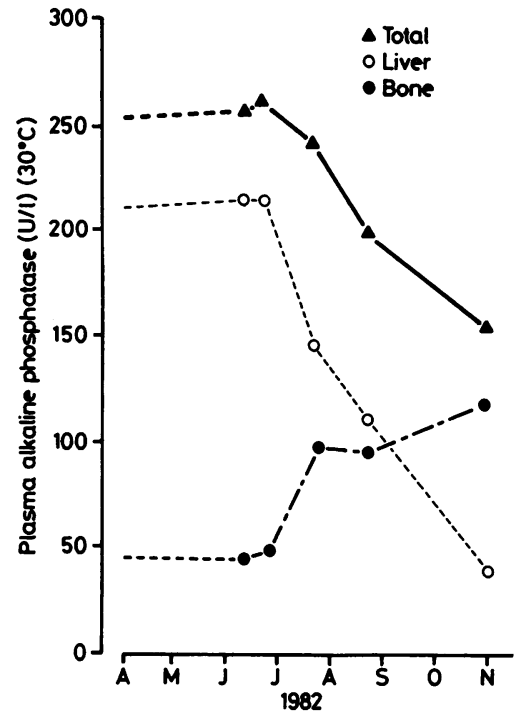

Fig 3 Serial changes in plasma total, bone, and liver alkaline phosphatase in case 20.

Plasma bone alkaline phosphatase activity correlated with the clinical symptomatology and with the radiographic findings in 14 of the 20 patients who were followed up serially. In five patients additional information relevant to patient management was obtained when plasma alkaline phosphatase isoenzyme data were made available. In two patients non-malignant disease was associated with an increase in isoenzyme activity. Examples are outlined briefly.

Case 20 (born 1931) (fig 3) Breast cancer was diagnosed in 1978. In March 1981 symptomatic bone metastases were diagnosed and bilateral oophorectomy was carried out, followed by a subjective and objective response. She developed recurrent bone pain in April 1982. Progressive bone metastases were shown and she was started on hormone therapy. She was first studied in June 1982 when she remained symptomatic with bone pain and tenderness over her right hip and pelvis. Bone alkaline phosphatase activity was considerably increased, but total alkaline phosphatase activity was only slightly increased. Liver isoenzyme activity was normal, as was an ultrasound scan of the liver. The following month she had radiotherapy to her pelvis. By November, although she still had extensive bone disease on bone scintigram, there had been a noticeable clinical improvement paralleled by a decline of total and bone isoenzyme activity to normal. Liver alkaline phosphatase activity had, however, shown a progressive increase and was abnormal. The liver was now just

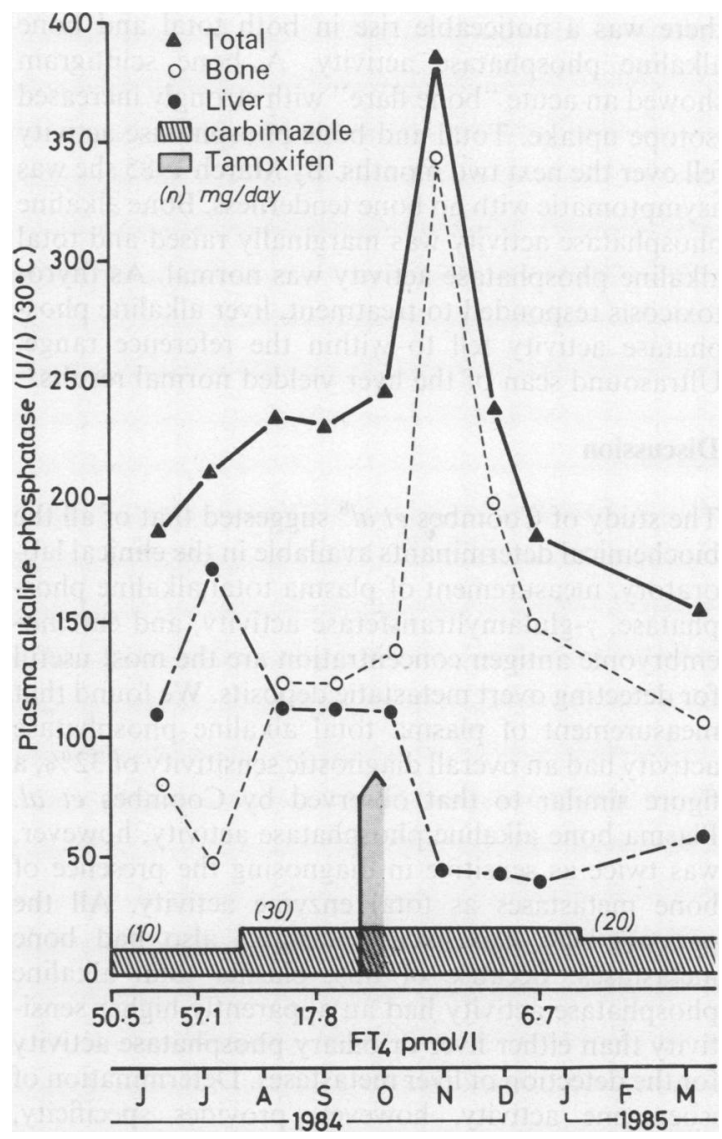

Fig 4 Serial changes in plasma total, bone, and liver alkaline phosphatase in case 5.

palpable below the costal margin and the ultrasound scan was compatible with liver metastases.

Case 5 (born 1909) (fig 4) Breast cancer was diagnosed in 1968. She had a history of gallstones which were treated medically between 1980 and 1982 . Thyrotoxicosis was diagnosed in March 1984, and she was started on carbimazole, $10 \mathrm{mg}$ daily. At the start of the study in June 1984 bone metastases were diagnosed using bone scintigraphy. Liver alkaline phosphatase activity was increased, total and bone phosphatase activities were initially within reference limits, but became abnormal in August 1984. A bone scan at that time showed generalised uptake, "compatible with metabolic bone disease", possibly associated with thyrotoxicosis. In addition, focal areas of increased uptake were suspicious of metastases. Bone metastases were confirmed on radiology and she was started on tamoxifen in October 1984. She developed acute bone pain and tenderness over the lower spine and pelvis (clinical "bone flare"). Within two weeks 
there was a noticeable rise in both total and bone alkaline phosphatase activity. A bone scintigram showed an acute "bone flare" with strongly increased isotope uptake. Total and bone phosphatase activity fell over the next two months. By March 1985 she was asymptomatic with no bone tenderness. Bone alkaline phosphatase activity was marginally raised and total alkaline phosphatase activity was normal. As thyrotoxicosis responded to treatment, liver alkaline phosphatase activity fell to within the reference range. Ultrasound scan of the liver yielded normal results.

\section{Discussion}

The study of Coombes $e t a l^{8}$ suggested that of all the biochemical determinants available in the clinical laboratory, measurement of plasma total alkaline phosphatase, $\gamma$-glutamyltransferase activity, and carcinoembryonic antigen concentration are the most useful for detecting overt metastatic deposits. We found that measurement of plasma total alkaline phosphatase activity had an overall diagnostic sensitivity of $32 \%$, a figure similar to that observed by Coombes et al. Plasma bone alkaline phosphatase activity, however, was twice as sensitive in diagnosing the presence of bone metastases as total enzyme activity. All the patients who had liver metastases also had bone metastases. Because of this, plasma total alkaline phosphatase activity had an apparently higher sensitivity than either liver or biliary phosphatase activity for the detection of liver metastases. Determination of isoenzyme activity, however, provides specificity, indicating the tissue of origin of the raised plasma total alkaline phosphatase activity. Overall, 45 of the combined total of 100 metastatic deposits were identified by alkaline phosphatase isoenzyme analysis compared with the 24 which showed total alkaline phosphatase elevation $(p<0.01)$.

We rarely observed an increase in both bone and liver alkaline phosphatase activity, even in those patients with clinical evidence of metastases at both sites. Additionally, occasional patients with a massive increase of one fraction showed complete absence of the other. This raises the possibility that production or post-translational modification (glycosylation) of one isoenzyme form interferes with that of the other, or that an excess of one form interferes with the separation procedure, though we have not observed this in non-malignant disease. Posen and Grunstein ${ }^{9}$ claimed that there is no evidence of a control mechanism whereby a circulating alkaline phosphatase activity can change the rate of production of the enzyme by different tissues or their removal from plasma. It is noteworthy, however, that in a diagram by Moss illustrating selective heat inactivation applied to the measurement of alkaline phosphatase isoenzymes in patients with malignant disease, 12 of 87 subjects (14\%) with increased liver phosphatase activity showed virtual absence of the bone fraction. ${ }^{10}$ If it is confirmed that an increase in one fraction interferes with the production or measurement of another in patients with malignancy, this would radically affect the interpretation of isoenzyme data, though in our serial studies (figs 3 and 4) correlation of changes in each fraction and clinical state was evident. Further studies are currently in progress to clarify the importance of this observation.

In contrast to previous findings made by ourselves and others, which have shown a higher prevalence of abnormalities of plasma biliary alkaline phosphatase activity, 1112 this fraction was detected in only nine of 25 patients with liver metastases. The reason for the low diagnostic sensitivity in this study is unclear. It may be due to earlier diagnosis of liver metastases in comparison with other studies, as special attention, using ultrasound or computed tomography at regular follow up, was given to their detection. Specificity of biliary alkaline phosphatase activity for liver disease was high, with a false positive rate of only $3 \%$ in patients with breast cancer with no liver metastases. We did not detect any placental like or Regan alkaline phosphatase activity by electrophoresis in the patients studied. These fractions have been reported in up to $10 \%$ of cancer patients ${ }^{13}$ but more sensitive immunological procedures are generally required for their detection. 10

Plasma $\gamma$-glutamyltransferase activity was the most sensitive diagnostic marker for the presence of liver metastases. Although increased in $87 \%$ of patients with liver metastases, it was also increased in $25 \%$ of patients without evidence of such metastases and consequently has a relatively low tissue specificity in cancer patients.

When enzyme or isoenzyme activity is abnormal, non-malignant causes should be excluded before more sophisticated investigations are performed to confirm the diagnosis of metastases. In case 5 liver alkaline phosphatase activity was raised at the time of initial investigation. Liver metastases were not present but thyrotoxicosis had been diagnosed three months previously. As thyrotoxicosis responded to treatment with carbimazole, liver alkaline phosphatase activity fell to within the reference range. Increased liver alkaline phosphatase activity in plasma is well recognised in hyperthyroid patients and was observed by Rhone et al in $73 \%$ of 42 hyperthyroid women. ${ }^{14}$

Intraindividual variation of plasma alkaline phosphatase activity is significantly less than population (interindividual) variation. Consequently, the upper limit of a population reference range (even that derived from a population with benign breast disease) 
is relatively insensitive as a diagnostic "cut off" limit to detect a pronounced increase in enzyme activity. Serial monitoring, with the detection of changes outside normal physiological variation, provides an earlier indication of disease. Serial analysis was used by Winkel et al to predict the occurrence of local and metastatic deposits in patients with breast cancer, ${ }^{15}$ but plasma total alkaline phosphatase and $\gamma$-glutamyltransferase activities were reported to provide no additional information. These workers, however, studied only 10 cases serially, and only six of these developed bone metastases; the remaining subjects developing metastases in soft tissue. We have not found these enzymes useful in the detection of soft tissue recurrence (normal in all of 15 patients studied), but as indicated above, this does not apply to the detection of organ metastatic disease. Additionally, isoenzyme determination provides superior diagnostic sensitivity and organ specificity to the measurement of total alkaline phosphatase activity.

A biochemical test that is used to diagnose and monitor a disease must provide additional information that is not readily available from clinical examination. Enzyme activity correlated with the clinical condition in most of our patients followed up serially, and plasma bone alkaline phosphatase activity was higher in the patients with symptomatic bone metastases. Serial isoenzyme analyses, by identifying metastases at unsuspected sites and prior to symptoms, gave additional information that would have been beneficial to patient management in $25 \%$ of patients. Consequently, serial analysis using plasma alkaline phosphatase isoenzymes and $\gamma$-glutamyltransferase determination for the detection of bone and liver metastases would seem to be justified. These procedures can be carried out at each outpatient visit, and the determinations can readily be carried out in the diagnostic laboratory with the methods used in this study.

We recommend serial measurement of bone alkaline phosphatase activity for the detection and monitoring of bone metastases, and liver alkaline phosphatase determination for liver metastases. Liver alkaline phosphatase activity should be combined with the more sensitive but less specific determination of $\gamma$-glutamyltransferase. Such measurements, though less sensitive than imaging procedures, can assist in screening for, and in the early detection of, a consid- erable proportion of metastases and can provide useful objective evidence of their response to treatment.

\section{References}

1 Cho SY, Choi HY. Causes of death and metastatic patterns in patients with mammary cancer. Am $J$ Clin Pathol 1980;73:232-4.

2 Roberts JG, Gravelle IH, Baum M, Bligh AS, Leach KG, Hughes LE. Evaluation of radiography and isotopic scintigraphy for detecting skeletal metastases in breast cancer. Lancet 1976;i:237-9.

3 Perez DJ, Powles TJ, Milan J, et al. Detection of breast carcinoma metastases in bone: relative merits of $x$ rays and skeletal scintigraphy. Lancet 1983;ii:613-6.

4 Sahm DF, Murray JL, Munson TL, Nordguist RE, Lerner MP. Gamma-glutamyltranspeptidase levels as an aid in the management of human cancer. Cancer 1983;52:1673-8.

5 Rosalki SB, Foo AY. Two new methods for separating and quantifying bone and liver alkaline phosphatase isoenzymes in plasma. Clin Chem 1984;30:1182-6.

6 Rosalki SB. Association of Clinical Biochemists' Working Party recommendations: proposed methods for determinations of some enzymes in blood serum. Association of Clinical Biochemists News Sheet 1980;Supplement:9-12.

7 Vecchio RJ. Predictive value of a single diagnostic test in unselected population. New Engl J Med 1966;274:1171-3.

8 Coombes RC, Powles TJ, Gazet JC, et al. Assessment of biochemical tests to screen for metastases in patients with breast cancer. Lancet 1980;i:296-8.

9 Posen S, Grunstein HS. Turnover rate of skeletal alkaline phosphatase in humans. Clin Chem 1982;28:153-4.

10 Moss DW. Alkaline phosphatase isoenzymes. Clin Chem 1982;28:2007-16.

11 Karmen C, Mayne PD, Foo AY, Parbhoo S, Rosalki SB. Measurement of biliary alkaline phosphatase by mini-column chromatography and by electrophoresis and its application to the detection of liver metastases in patients with breast cancer. $J$ Clin Pathol 1984;37:212-7.

12 Viot M, Joulin C, Cambon P, Krebs BP, Schneider M, Lelanne CM. The value of serum alkaline phosphatase $\alpha_{1}$ isoenzyme in the diagnosis of liver metastases. Biomédicine 1979;31:74-7.

13 Stolbach LL, Krant MJ, Fishman WH. Ectopic production of an alkaline phosphatase isoenzyme in patients with cancer. New Engl J Med 1969;282:757-62.

14 Rhone DP, Berlinger FS, White FM. Tissue sources of elevated serum alkaline phosphatase activity in hyperthyroid patients. Am J Pathol 1970;74:381-6.

15 Winkel P, Bentzon MW, Statland BE, Mouridsen H, Skeike O. Predicting recurrence in patients with breast cancer from cumulative laboratory results: a new technique for the application of time series analysis. Clin Chem 1982;28:2057-67.

Requests for reprints to: Dr SB Rosalki, Consultant in Chemical Pathology and Human Metabolism, Royal Free Hospital, Pond Street, Hampstead, London NW3 2QG, England. 